\title{
Multimodal fusion system for NDT and Metrology
}

by B. Verney*, M. Akhloufi* and O. Aubreton**

* Center of Robotics and Vision (CRVI), 205 rte. Mgr. Bourget, G272,Lévis, Québec,G6V 6Z9, Canada, moulay.akhloufi@crvi.ca, benjamin.verney@crvi.ca

** Laboratoire Le2i, UMR CNRS 6306, 12 rue de la fonderie, 71200 Le Creusot, France, olivier.aubreton@u-bourgogne.fr

\begin{abstract}
3D scanning and modeling is used in a wide range of applications like manufacturing, aerospace, security, biomedicine, etc. This kind of systems allows the scanning of a 3D point cloud that can be meshed and rendered in order to obtain a complete 3D model. However, 3D vision cannot detect subsurface defects. This later can be achieved using other imaging modalities, like thermal infrared imaging. This modality is very popular in non-destructive testing and visual inspection. In this work, we present a multi-modal fusion framework for combining these two modalities. We present an approach for a robotic inspection system integrating the proposed framework for 3D-NDT multimodal control and analysis.
\end{abstract}

\section{Introduction}

Today, quality control plays an increasingly important role in many industrial fields. This control can be used for surface finish inspection, material quality and object structure. The quality of an object can be quantified in many ways and by various processes. Among them, non-destructive testing (NDT) represent a good alternative when we want to avoid any object degradation during the measurement process [1-2]. In this paper, we use two non-destructive control methods: 3D digitization and Infrared Thermography. 3D vision for metrology and inspection [3-4] is largely used by industries in aerospace, manufacturing, mining, etc. It allows the inspection of different kinds of products for metrological inspection and the 3D detection of visible defects. The 3D data can be compared to CAD models using 3D matching techniques in order to insure that the product respects the defined tolerances.

However, 3D vision is unable to detect hidden defects. This can be achieved using other imaging modalities, like thermal infrared imaging [1-2]. With the decreasing price of infrared cameras, thermal infrared imaging is becoming very popular in non-destructive testing. These two modalities (3D and infrared) are used individually for the purpose of quality control in many applications. The same object can be inspected with the two approaches leading to two individual analysis during the same quality control and visual inspection (e.g. composite materials in aerospace products).

In this work, we present a new architecture for 3D and thermal data fusion in order to improve the process of visual inspection of industrial products. The proposed approach uses thermal diffusion images fused with 3D models in order to model non-visible structure defects in a three-dimensional space.

\section{System architecture}

Figures 1 shows the proposed framework and the architecture for the fusion of 3D and thermal infrared data for visual inspection [5].

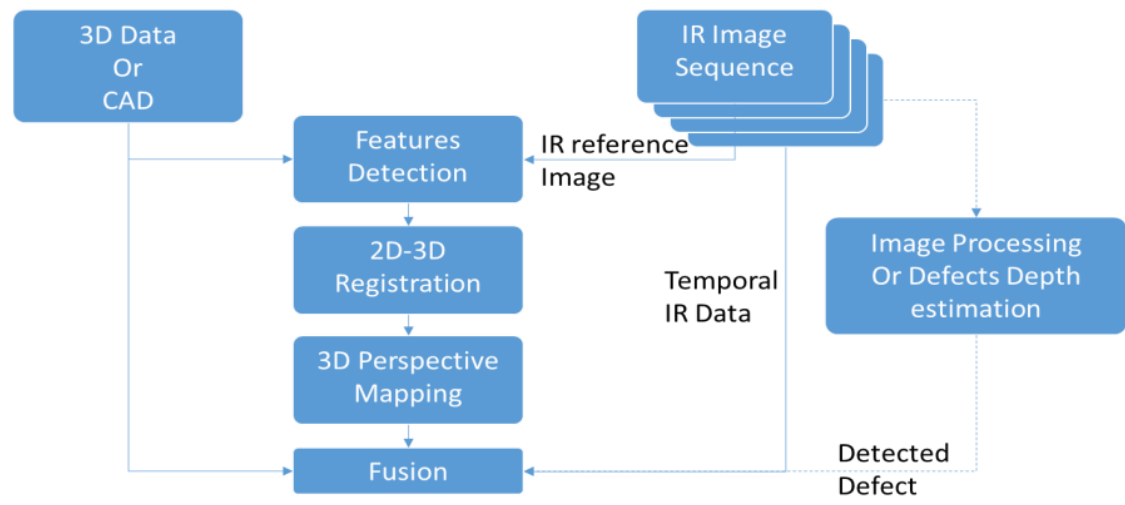

Fig. 1. Architecture of the multimodal fusion framework 
The proposed architecture involves six steps:

1. 3D image acquisition: a 3D camera is used in order to digitize the object and record the 3D point cloud. This point cloud in then meshed and rendered in order to build a complete 3D model of the digitized object. The resulting 3D images can be used for 3D inspection, 3D metrology, CAD matching, etc.

2. Thermal Infrared sequence capture: an infrared sequence is continuously captured while a heating source is used in order to heat the surface under inspection.

3. Feature detection: in order to register the $3 \mathrm{D}$ and the $2 \mathrm{D}$ infrared images we need to compute the transformation matrix between them. Feature detection approaches can be used at this step. In this work we used Hough transform [6] in order to detect salient features from the 3D image and an infrared reference image.

4. 2D-3D registration: the extracted features are used to compute the transformation between the 2D and $3 \mathrm{D}$ object features. A K-Nearest Neighbors process [7] is performed in order to associate corresponding features and compute the transformation matrix.

5. 3D perspective mapping: the registered data are used to extract the 3D perspective mapping between the sequence of infrared images and the 3D model.

6. Multimodal Fusion: the obtained mapping is applied in order to fuse pairs of "3D-2D infrared images". The following can be performed:

a. Fusion of the temporal thermal data (thermograms) and the 3D image (or 3D CAD);

b. Fusion of the detected defect in the thermal image and the 3D image (or 3D CAD);

c. Fusion of the 3D-CAD matching result and the thermal data (thermograms or defects).

The proposed architecture is modular and designed to adapt to any kind of 3D and infrared imaging systems.

\section{3D Vision}

3D vision is an area of computer vision that has been widely studied [3-4, 8-9]. A large number of methods have been proposed for this purpose. They can be divided into active and passive range sensing methods. Passive range scanning techniques do not influence the scene lighting. Stereovision is a passive technique used in applications where the precision of the captured data is less important [10] (fire tracking, pedestrian and vehicles tracking, face modeling, etc.). In industrial applications, the most popular techniques use active strategies that control the lighting in order to build a more precise 3D model (laser triangulation, phase shifting, moiré interferometry, structured light projection, etc.) Laser triangulation is the most popular approach and many commercial products are available. They can easily produce high precision results.

In this work we use an industrial laser triangulation system from SICK [11]. In order to build the 3D model, the camera (or the product) must be moved to create a set of profiles (Figure 2) and build the 3D model. The captured 3D point cloud is then meshed and rendered in order to obtain the complete 3D model (Figure 3). This 3D data can be analyzed in order to extract the surface defects, perform metrological analysis, etc.

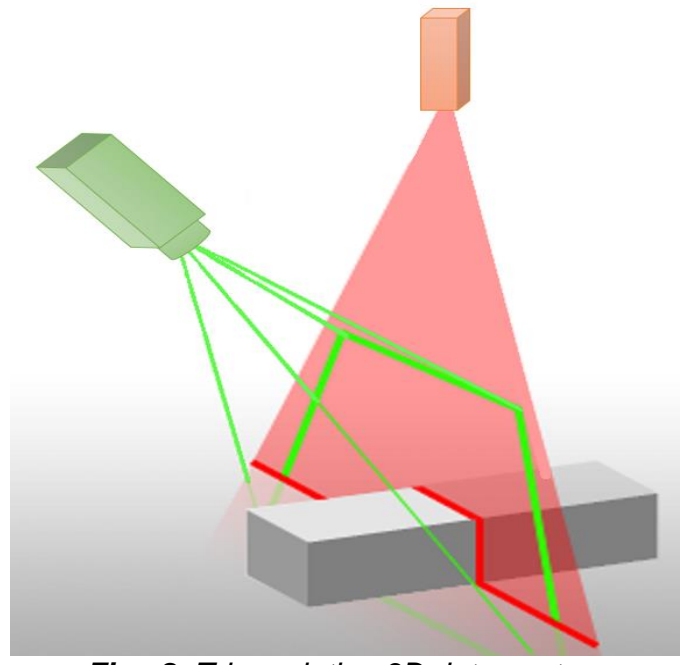

Fig. 2. Triangulation $3 D$ data capture 


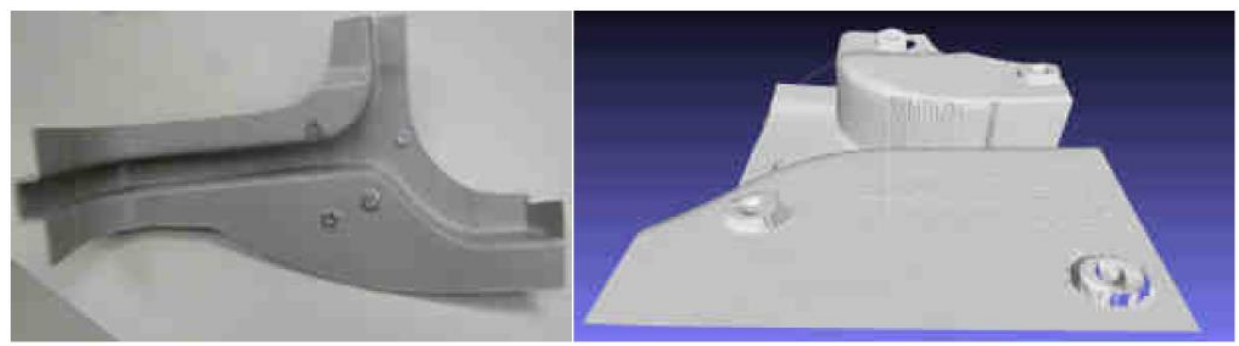

Fig. 3. 3D modeling (left: original part, right: rendered 3D scanned data)

\section{Infrared Thermal Imaging}

Infrared imaging is becoming an important area of research in computer vision. This is mainly because of the decreasing price of infrared cameras and their availability. Infrared imaging is widely used in defense and security applications [12], and NDT. More recently, new applications of infrared are emerging: fire propagation study [13], face recognition [14], hand biometrics [15], biomedical applications [16], etc.

The infrared spectrum span the bands of $0.74 \mu \mathrm{m}$ to $1000 \mu \mathrm{m}$ of the electromagnetic spectrum (Figure 4) [17]. The most popular spectral bands in infrared imaging applications are: Near IR $(0.74-1 \mu \mathrm{m})$, Short-wave IR $(1-3 \mu \mathrm{m})$, Midwave IR $(3-5 \mu \mathrm{m})$ and Long-wave IR $(8-14 \mu \mathrm{m})$. Near IR and Short-wave IR are said active infrared spectrums and capture the reflectivity of incident lights on the surface. Mid-wave IR and Long-wave IR are said thermal infrared spectrums and capture the emitted thermal radiation from the imaged object. These two last thermal spectrums are widely used for imaging non-visible defects in NDT applications.

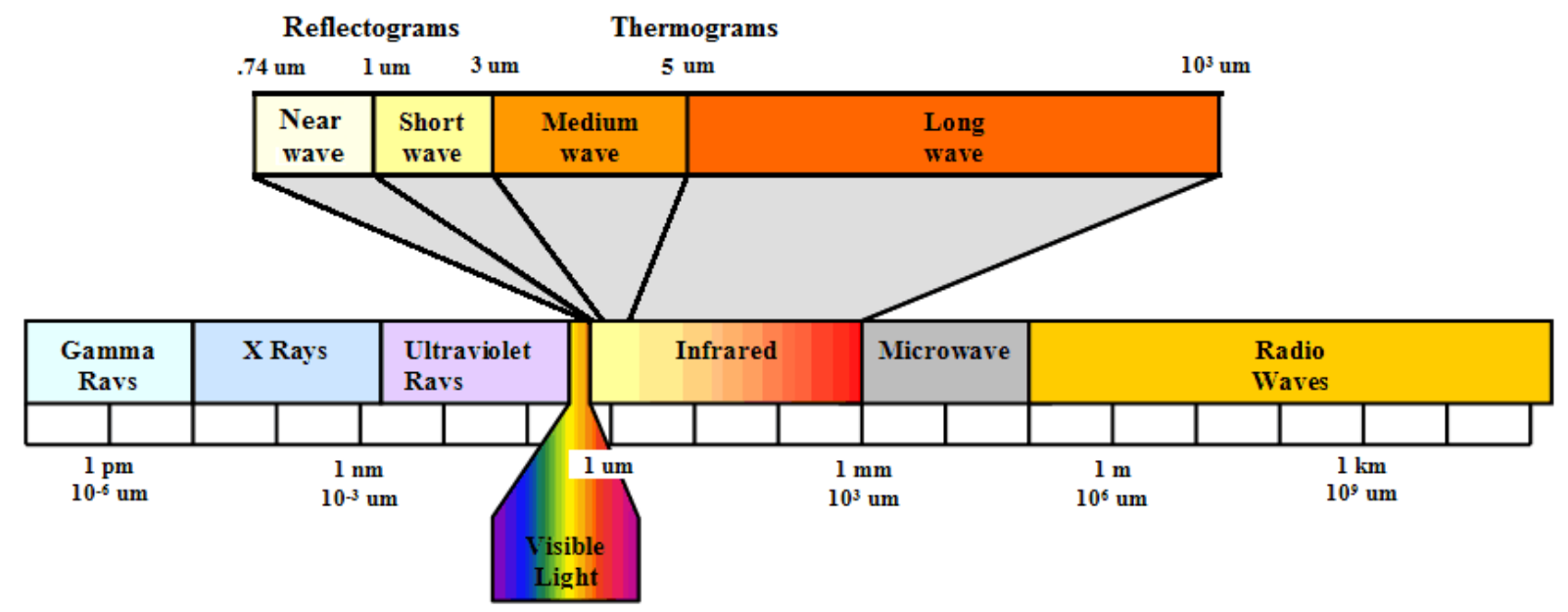

Fig. 4. Electromagnetic spectrum showing the infrared interval

Many thermal infrared techniques are used in NDT. The most popular and efficient are active thermography techniques [2]. They use external excitation sources in order to induce thermal radiation from the inspected object and detect subsurface defects. The excitation sources can be optical (pulsed and lock-in thermography), mechanical (lock-in and burst vibrothermography) or electromagnetic (pulsed eddy current thermography). Many of them can work in either transmission or reflection mode.

In this work, we use pulsed thermography technique. The process of pulsed thermography sends a pulse of a certain length in time. This pulse is a heating source that acts on the inspected object. The thermal radiations of the defects are different and become visible over time. In transmission mode, the object is positioned between the heating source and the infrared camera. The result is a thermal image sequence (thermogram). Image processing techniques can be applied to the thermogram in order to extract the subsurface defects. Figure 6 gives an example of a thermal image captured in a Long-wave infrared spectrum (LWIR). A PV-320 camera from Electrophysics was used in our experiments [18]. 


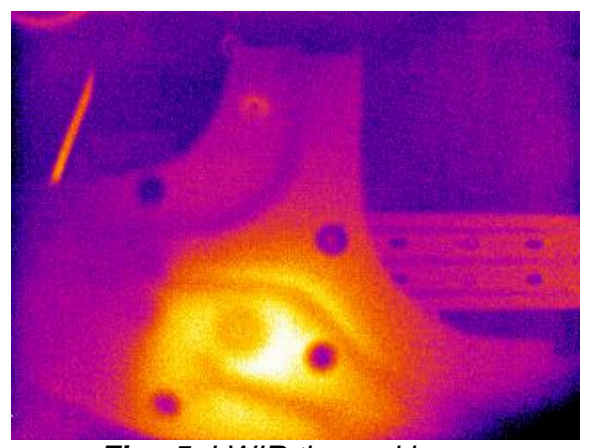

Fig. 5. LWIR thermal image

\section{Feature detection and registration}

A Hough transform was used to detect salient features in the 3D image and the 2D infrared images to perform the registration.

The Hough Transform is a commonly used algorithm for detecting shapes in an image. This technique is based on the original Hough Transform algorithm used for detecting an object having a simple analytic equation describing its boundary. Hough transform is used for detecting lines, circles or ellipses.

GHT (Generalized Hough Transform) [6] is an extension of the standard Hough that supports object boundaries of arbitrary non-analytic shape. Instead of using a parametric equation, GHT uses a look-up table to define the relationship between the boundary positions and orientations and the Hough space parameters. In order to detect an object a model of this objet is learned and a look-up table values computed offline.

For example, suppose that we know the shape and orientation of the desired feature (Figure 6). We can specify an arbitrary reference point (Xref, Yref) within the feature, with respect to which the shape (i.e. the distance $r$ and angle $\beta$ of normal lines drawn from the boundary to this reference point) of the feature is defined. Our look-up table (i.e. R-table) will consist of these distance and direction pairs, indexed by the orientation $\Omega$ of the boundary.

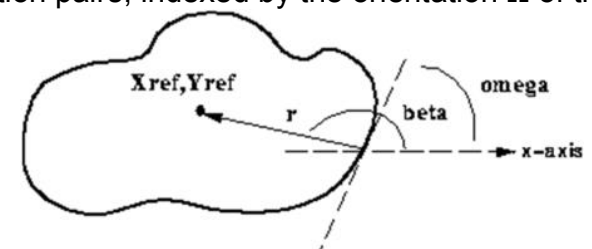

Fig. 6. R-T́able components

The Hough transform space is now defined in terms of the possible positions of the shape in the image, i.e. the possible ranges of (Xref, Yref). In other words, the transformation is defined by Eqs. (1) and (2):

$$
\begin{aligned}
& x_{r e f}=x+r^{*} \cos (\beta) \\
& y_{r e f}=y+r^{*} \sin (\beta)
\end{aligned}
$$

(The $r$ and $\beta$ values are derived from the R-table for specific known orientations $\Omega$ ). If the orientation of the desired feature is unknown, this procedure is complicated by the fact that we must extend the accumulator by incorporating an extra parameter to account for changes in orientation.

In our case, the features are circles of small scale changes so we do not need to account for the variation in orientation. This helps reducing the scale search in the Hough space and makes the procedure more robust.

Feature detection is performed after an edge detection on the $3 \mathrm{D}$ and the infrared images. In the 3D case, the height map of the 3D image is used. Figure 7, shows the process of edge and circle detection in the height map and the infrared image. 


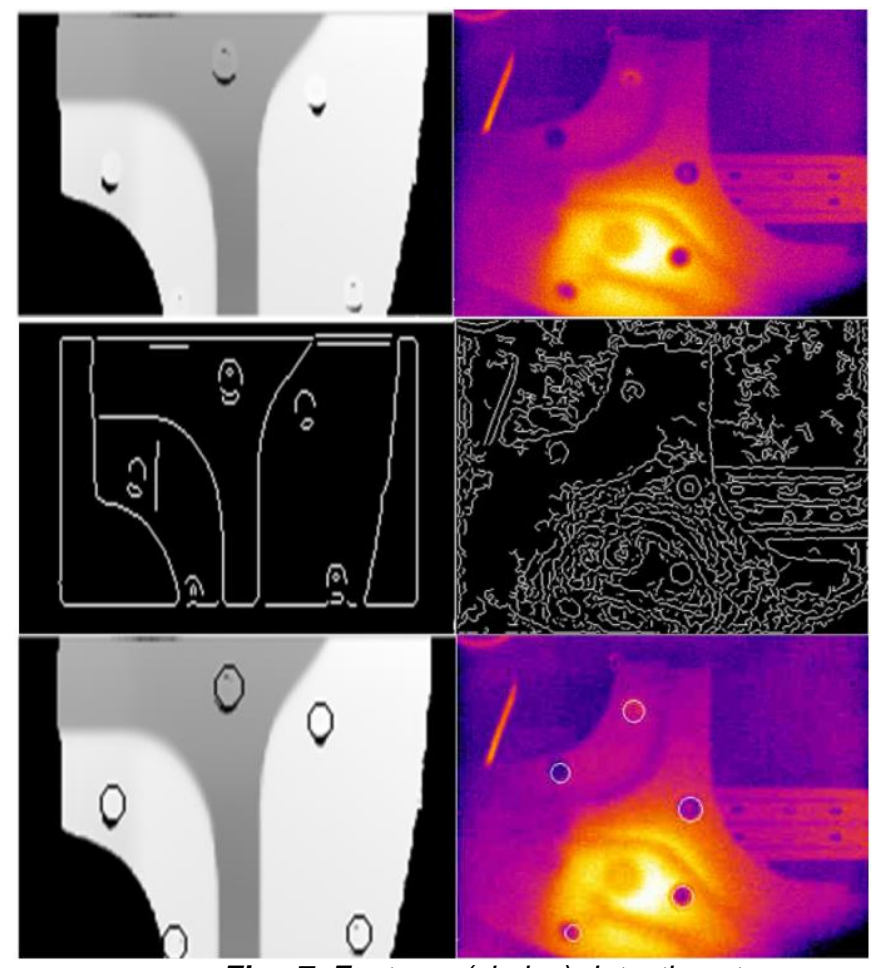

Fig. 7. Features (circles) detection steps

Once the feature detection is performed in the 3D and infrared images, a k-nearest neighbor algorithm (k-NN) [7] is applied in order to pair matching features in the two modalities (Figure 8). This procedure allows the extraction of the registration matrix between the 3D and the infrared images.

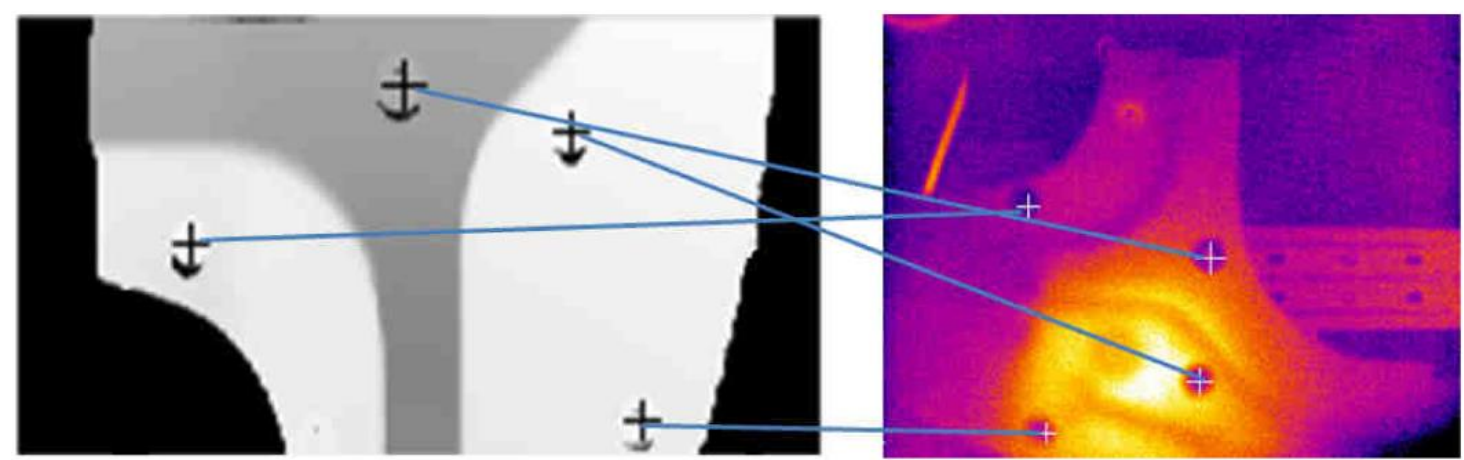

Fig. 8. K-NN features matching

\section{Perspective mapping and fusion}

The steps above allows the extraction of the transformation matrix between the 3D and the 2D infrared images. This matrix is used for perspective mapping in 3D space. An over determined system is used to solve for the perspective mapping using a linear system of equations [19-20].

Perspective correct texture mapping accounts for the vertices positions in 3D space. This is achieved by dividing the coordinates by their depth (Instead of interpolating the coordinates directly). The reciprocal of the depth value is also interpolated and used to recover the perspective coordinate [19-20].

The results allows to fuse the obtained thermogram with the 3D image in 3D space using texture mapping techniques [19-20]. 


\section{Experimental results}

Figure 9 shows the experimental setup containing the Electrophysics PV-320 infrared camera operating in the LWIR spectrum, and a Sick IVP 3D Laser Triangulation camera. The 3D camera is synchronized with a linear axis for scanning purposes.

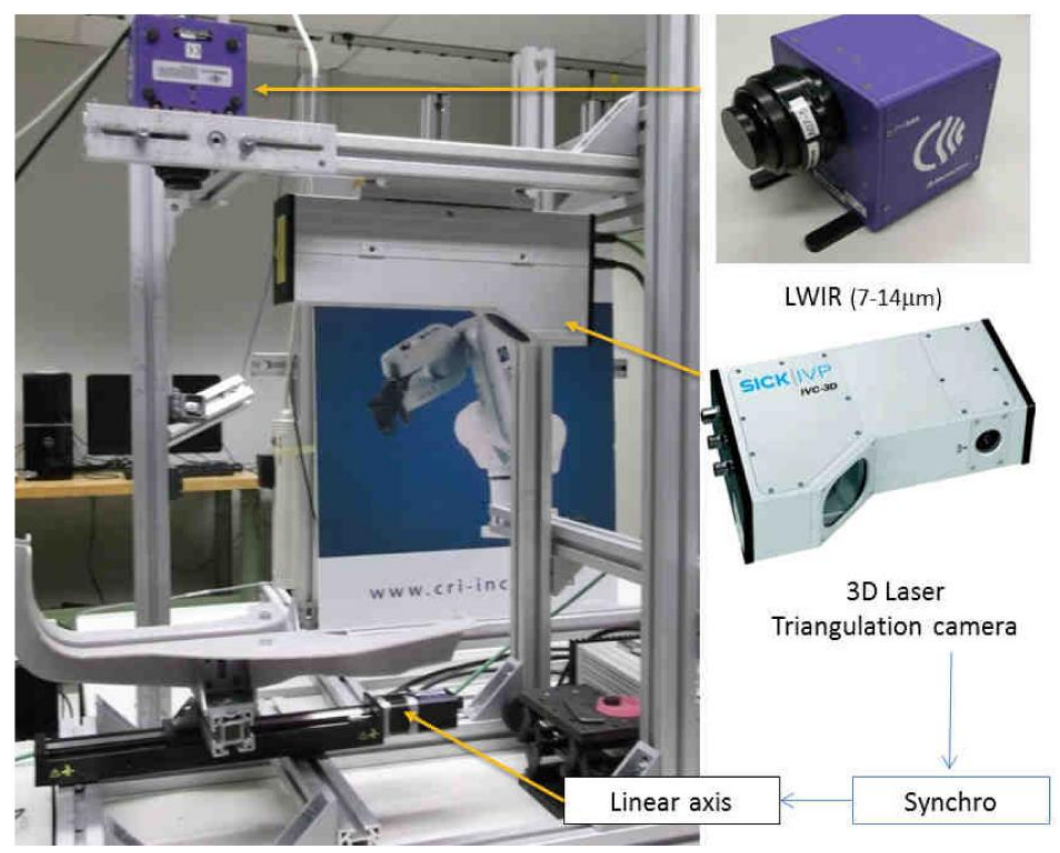

Fig. 9. Experimental setup

Figure 10 shows two examples of result by the fusion scheme. A 3D mapped thermogram is used for visualizing the subsurface defects in two types of materials (Composites in the upper image and PVC in the lower image of Figure 10).

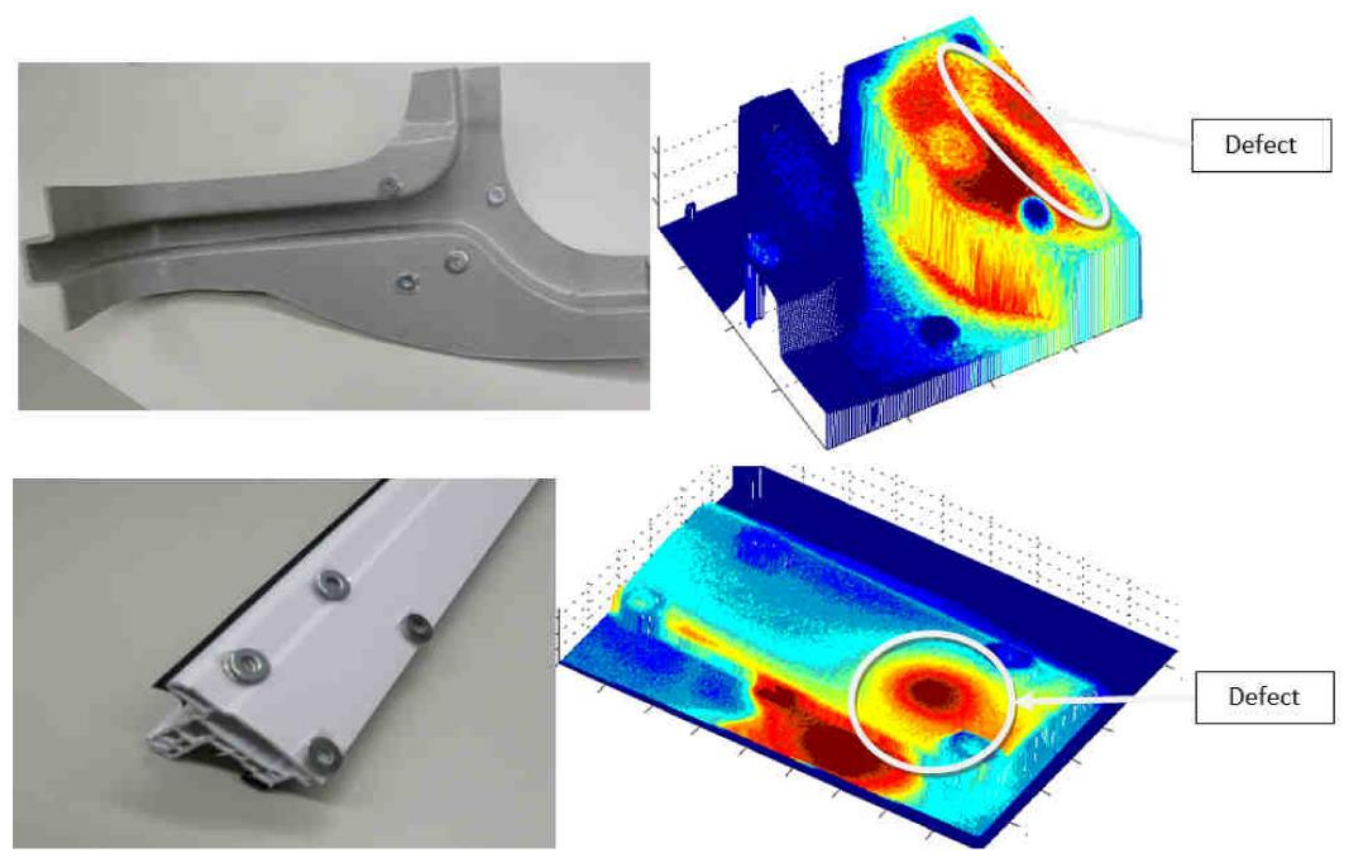

Fig. 10. Example of fused thermogram in $3 D$ space and defect visualization 
The 3D fused thermogram can be moved in 3D space in order to visualize different point of views. (Figure 11)
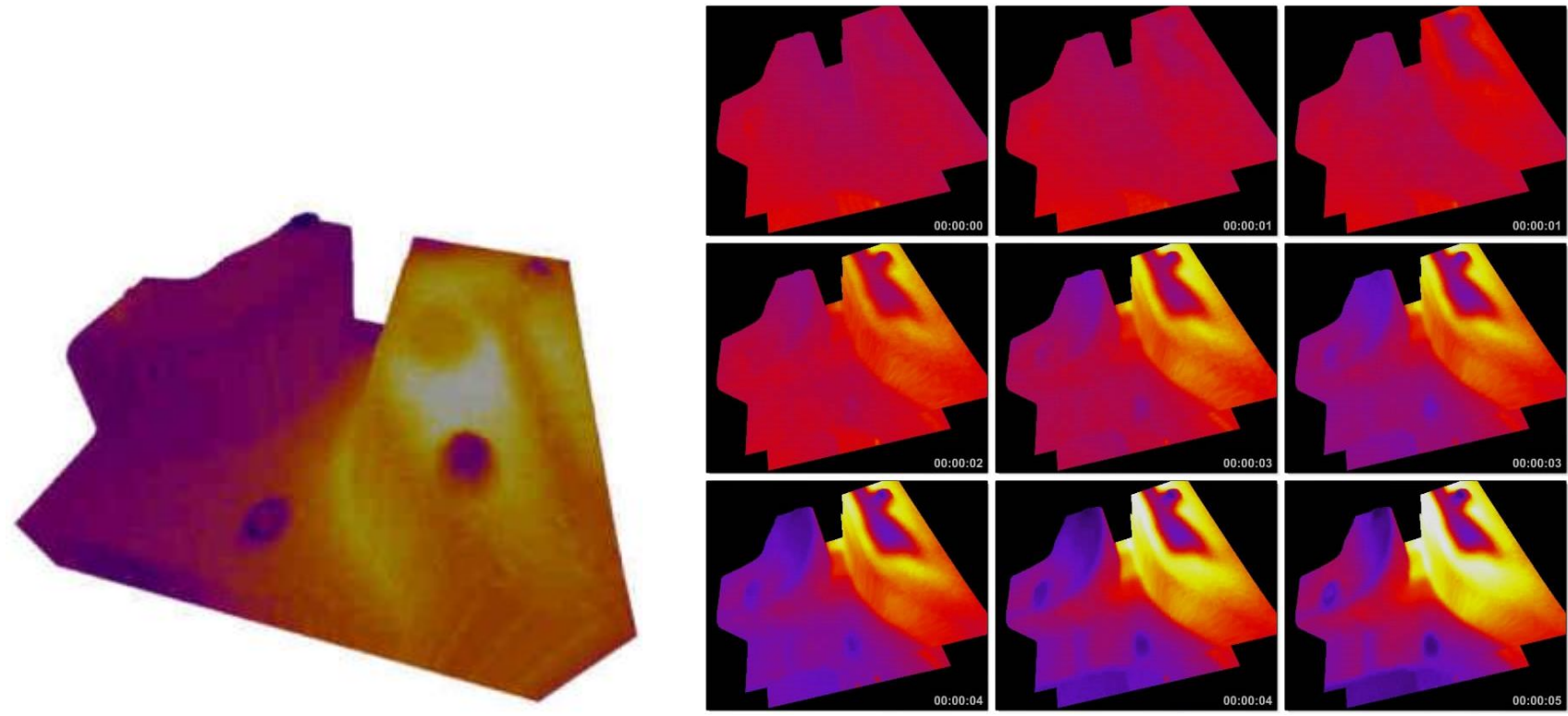

Fig. 11. A $3 D$ fused multimodal thermogram view

In some cases, we need to visualize the detected defect without displaying the complete thermogram. Two options are available. We can visualize the defect position over a fused infrared image (the image that illustrates mostly the defect in the thermogram) (Figure 12). Also, we can only show the position of the defect over the 3D mapped data in 3D space (Figure 12). We can also visualize the defect form obtained after image processing on the same images above or on the CAD model.
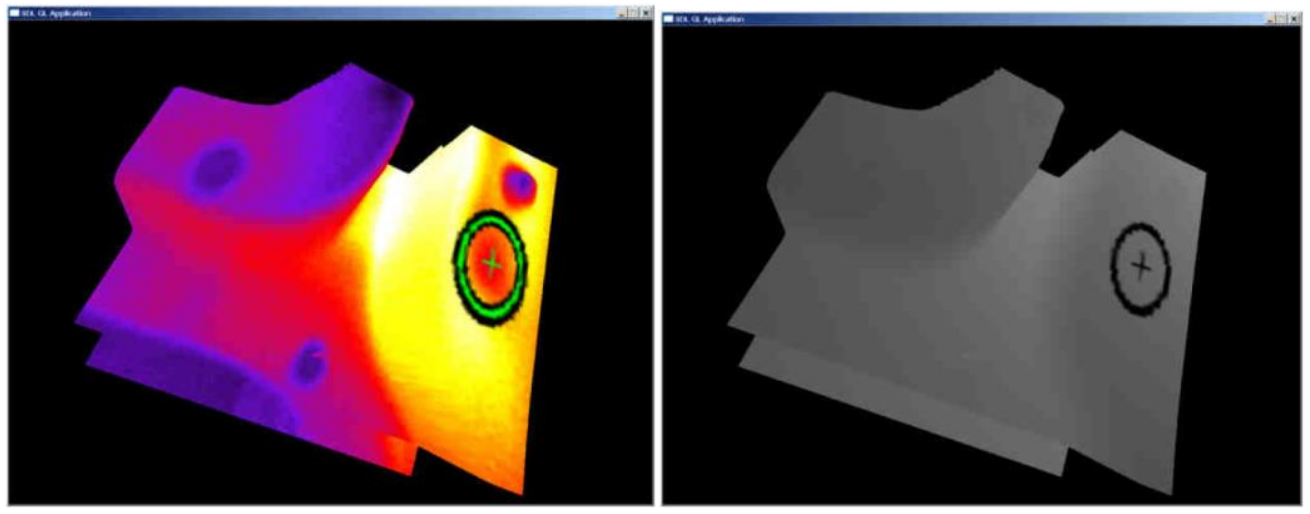

Fig. 12. Defect position on an infrared image in $3 D$ space and on a $3 D$ mapped in $3 D$ space

\section{Conclusion}

This work presents a new architecture for fusing 3D and thermal data in order to conduct 3D inspection and NDT. With the proposed framework, we can visualize 3D metrological information and subsurface defects obtained by NDT techniques (e.g. thermography).

The proposed framework is modular and can be adapted easily to other types of technologies. We can add other 3D camera setups like active stereo systems, phase shifting cameras, etc. In the NDT part, we can accommodate different types of thermography techniques and other technologies like Laser ultrasound. Plugin-based flexible software architecture was designed for this purpose. Future work includes the integration of this approach in a robotic 3D-NDT inspection system. Figure 13 shows the simulation of this robotic environment. 


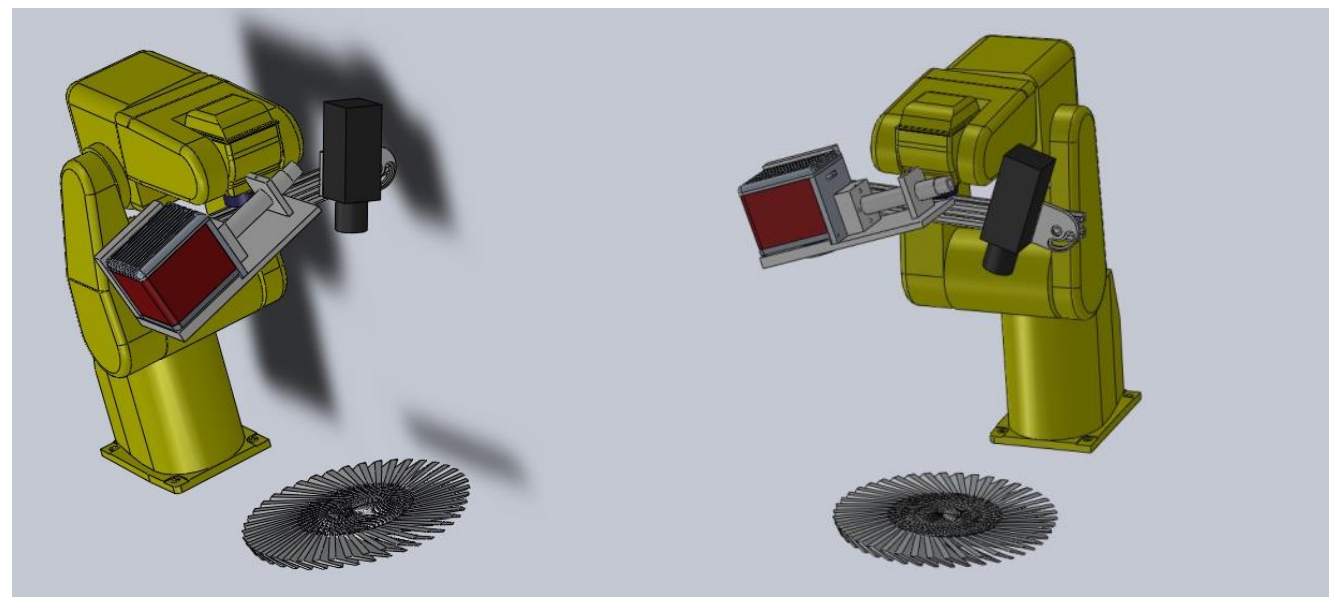

Fig. 13. Robotic 3D-NDT inspection system

\section{REFERENCES}

[1] NDT Resource Center, "Introduction to NDT," http:// www.ndted.org/GeneralResources/IntroToNDT/GenIntroNDT.htm, visited March 2013.

[2] Maldague, X., "Introduction to NDT by Active Infrared Thermography," Materials Evaluation, 6[9]: 10601073, 2002.

[3] Trucco, E. and Verri, A., "Introductory Techniques for 3D Computer Vision," Prentice-Hall, 1998.

[4] Zhang, S., "Handbook of 3D Machine Vision: Optical Metrology and Imaging," Series in Optics and Optoelectronics by Taylor \& Francis, USA, March 15, 2013.

[5] Akhloufi, M.A., and Verney, B., "Fusion framework for 3D inspection and thermal NDT", SAE 2013 AeroTech Congress, Manufacturing/Materials/Structures - Metrology Automated Systems (ATC907), September 24-26, 2013.

[6] Ballard, D.H., "Generalizing the Hough Transform to Detect Arbitrary Shapes," Pattern Recognition, Vol.13, No.2, p.111-122, 1981.

[7] Everitt, B. S., Landau, S., Leese, M. and Stahl, D. "Miscellaneous Clustering Methods," in Cluster Analysis, 5th Edition, John Wiley \& Sons, Ltd, Chichester, UK, 2011.

[8] Wöhler, C., "3D Computer Vision Efficient Methods and Applications," X media publishing Wöhler 2nd ed. 2013.

[9] Cyganek, B. and Siebert, J.P., "An Introduction to 3D Computer Vision Techniques and Algorithms," WileyBlackwell (an imprint of John Wiley \& Sons Ltd), February 2009.

[10] Scharstein, D. and Szeliski R., "A taxonomy and evaluation of dense two-frame stereo correspondence algorithms," Int. J. Com. Vis., 47(1/2/3): 7-42, 2002.

[11] Sick IVP, http://www.sick.com/ivp, visited March 2013.

[12] Akhloufi, M.A., Porcher, C., and Bendada, A., "Fusion of thermal infrared and visible spectrum for robust pedestrian tracking ", Proc. of SPIE, Airborne Intelligence, Surveillance, Reconnaissance (ISR) Systems and Applications XI, p. 9076-26, Baltimore, MD, May 2014.

[13] Rossi, L., Toulouse, T., Akhloufi, M.A., Pieri, A. and Tison, Y., "Estimation of spreading fire geometrical characteristics using near infrared stereovision," SPIE Proceedings Vol. 8650, Three-Dimensional Image Processing (3DIP) and Applications 2013, 86500A, 2013.

[14] Akhloufi, M.A. and Bendada, A., "Fusion of active and passive infrared images for face recognition," Proc. SPIE Volume 8705, Thermosense: Thermal Infrared Applications XXXV, 8705-10. 2013.

[15] Akhloufi, M.A. and Bendada, A., "Hand and Wrist Physiological Features Extraction for near Infrared Biometrics," Computer and Robot Vision, 2008. CRV '08. Canadian Conference on, vol., no., pp.341, 344, 28-30 May 2008.

[16] Ring, EF, and Ammer K. "Infrared thermal imaging in medicine," Physiol Meas. 33(3):R33-46. Feb 28, 2012.

[17] MIVIM, "Electromagnetic Spectrum," http://mivim.gel.ulaval.ca/dynamique/index.php?idD=58\&Lang1 2013, visited March 2013.

[18] Electrophysics "PV-320 infrared camera," http:// www.electrophysics.com/Browse/Brw_ProductLineCategory.asp?Categoryld=144\&Area=TG, visited March 2013.

[19] Wolberg, G., "Digital Image Warping", IEEE Computer Society Press, 1990.

[20] Foley, J.D. van Dam, A., Feiner, S.K. and Hughes, J.F., "Computer Graphics - Principles and Practice", Addison- Wesley, 1990. 